\title{
UCRL-CONF-218420
}

LAW RENCE LIVERMORE N A T IO N A L LABORATORY

\section{Recent Progress in Fermion Monte Carlo}

M. H. Kalos, F. Pederiva

January 26, 2006

Pacifichem 2005

Honolulu, HI, United States

December 15, 2005 through December 20, 2005 
This document was prepared as an account of work sponsored by an agency of the United States Government. Neither the United States Government nor the University of California nor any of their employees, makes any warranty, express or implied, or assumes any legal liability or responsibility for the accuracy, completeness, or usefulness of any information, apparatus, product, or process disclosed, or represents that its use would not infringe privately owned rights. Reference herein to any specific commercial product, process, or service by trade name, trademark, manufacturer, or otherwise, does not necessarily constitute or imply its endorsement, recommendation, or favoring by the United States Government or the University of California. The views and opinions of authors expressed herein do not necessarily state or reflect those of the United States Government or the University of California, and shall not be used for advertising or product endorsement purposes. 


\title{
Recent Progress in Fermion Monte Carlo
}

\author{
M. H. Kalos ${ }^{1}$ and F. Pederiva ${ }^{2}$ \\ ${ }^{1}$ Lawrence Livermore National Laboratory Livermore, CA USA \\ ${ }^{2}$ Dipartimento di Fisica, Università di Trento, Povo-Trento, Italy
}

\begin{abstract}
Our approach to the solution of the Schrödinger Equation for many-fermion systems has been extensively revised. We have devised a generalization of "acceptance/rejection" that applies to signed random walkers. We have introduced a new class of importance functions for two walkers that better reflects the structure of the enlarged Euclidean space of the pair. For greater flexibility, we no longer rely on the "local energy" of the importance function to determine the dynamics of the walk. We sketch these technical changes and give new results for the two-dimensional electron gas.
\end{abstract}

\section{Introduction}

This paper is a progress report on our research in Fermion Monte Carlo, which is aimed at developing a method for solving many-fermion problems that is efficient in the spirit of Monte Carlo methods, that is polynomial in particle number, and that uses no uncontrolled approximations.

The phrase "no uncontrolled approximations" is often used in a somewhat ambiguous way. We propose the following criteria:

\section{RESERVE THIS SPACE}


1. Any calculation should produce an error estimate. In a Monte Carlo calculation, the statistical error is not sufficient: what is required is an internal estimate of the difference between the expected value and the correct answer to the physical or mathematical model being solved.

2. The results should agree with known exact results or with reliable experimental data if available.

3. The computer time required to reduce the total error by some fraction should be a polynomial of low degree in the ratio of errors.

The criteria deserve further discussion. Criterion 2. can hardly be controversial; perhaps a reminder is needed that experimental results can be wrong or wrongly interpreted.

The desirability of criterion 3. is clear, but often ignored: many computations can be carried through to an apparently satisfactory level of agreement with other results, but can only be further improved at great cost, either because of an approximation essential to the computation, a combinatorial explosion in an expansion or a set of basis functions, or because of the inherent computational complexity of the method. Nevertheless, we restrict the term "no uncontrolled approximations" to methods that satisfy it.

Criterion 3. is clearly the most stringent. Many numerical calculationsand purely theoretical work as well-require physical, mathematical, or numerical approximations to be feasible. We do not assert that such calculations are invalid. Indeed, in the work described below and elsewhere we make two well-known approximations-- neglect of relativistic effects and fixing the nuclear positions. The effect of these can be accurately estimated. Our argument is that a numerical solution of the non-relativistic Schrödinger equation for a many-electron system that has no uncontrolled approximations would be a tremendously valuable asset to the theory of electronic structure. Since corresponding methods for the ground state of many-boson systems exist and have also proved valuable, it is natural to seek the analog for many-fermion systems.

It is the notorious "fermion sign problem" that makes the extension difficult. Perhaps another digression is worth while. If one defines a "sign problem" in Monte Carlo as the degradation of statistical efficiency because of the presence of negative signs, then there are many "sign problems." Some are provably intractable; some are clearly trivial or can be transformed away.

The sign problem that arises in treating the Schrödinger equation for manyfermion systems is not trivial, but it is not known to be intractable. 
The standard way to treat such systems within the framework of Quantum Monte Carlo is to impose the "fixed-node approximation," ${ }^{1}$ namely to choose an antisymmetric trial function and to follow walkers that diffuse only in domains in which that function does not change sign. If one removes the fixednode constraint, then the Monte Carlo estimates of the numerator and denominator of the energy quotient degenerate exponentially fast to zero with statistical errors that asymptotically dominate their mean values. This behavior reflects the fact that the distributions of the random walkers converge to that of the symmetric ground state in which the averages of antisymmetric test functions are zero.

The difficulty is not simply technical; it reflects some deep challenges. These can be characterized in several ways that may be clues about how to cure them.

One way of describing the problem is that the random walk that solves the Schrödinger equation is "local." All decisions about the dynamics are based on the position of a walker. But the Pauli principle is global in character; it connects the properties of a physically acceptable solution at distinct points usually well separated in configuration space. Reconciling the random walk with antisymmetry seems to require some action at a distance. In effect, this is what the fixed-node restriction does, at the cost of an uncontrolled approximation.

We introduce walkers with algebraic signs: a positive walker adds its contributions (which may have either sign) to numerator and denominator of the energy quotient. A negative walker subtracts its contributions. The use of such signed walkers does nothing in itself to avoid the asymptotic decay of signal to noise as described above. If these walkers diffuse independently then the distributions of both populations will approach that of the symmetric ground state. But there is now an opportunity to ameliorate that decay by changing the dynamics. Clearly, the two walkers must obey different dynamics; at the least they must follow different guiding functions. If not, then, in the long run the two populations will have the same distributions (corrected for the effect of importance sampling) namely that of the symmetric ground state.

Another opportunity for improvement lies in the possibility of canceling walkers of opposite signs against each other, while leaving unchanged the projections with antisymmetric test functions. While this is straightforward in principle, it is effective only when the walkers are close to each other, and close pairs become exponentially rare in high-dimensional space- i.e. for large particle numbers.

Correlating the dynamics of two random walkers makes it possible to cancel walkers efficiently even for many-body systems.

Our research ${ }^{3}$ has been motivated by the expectation that this sign problem can be solved in an effective way, using these and perhaps other techniques. 


\section{Importance functions}

Let $\varphi_{A}(\mathbf{R})$ and $\varphi_{S}(\mathbf{R})$ be approximate wave functions for the antisymmetric and symmetric ground states of our system. Now define

$$
\begin{aligned}
& \Phi_{G}^{ \pm}(\mathbf{R})=\sqrt{\varphi_{S}^{2}(\mathbf{R})+g_{1}^{2} \varphi_{A}^{2}(\mathbf{R})} \pm g_{0} \varphi_{A}(\mathbf{R})+g_{0}\left|\varphi_{A}(\mathbf{R})\right| \\
& \Phi_{S}(\mathbf{R})=\sqrt{\varphi_{S}^{2}(\mathbf{R})+g_{1}^{2} \varphi_{A}^{2}(\mathbf{R})} \\
& \Psi\left(\mathbf{R}^{+}, \mathbf{R}^{-}\right)=\Phi_{G}^{+}\left(\mathbf{R}^{+}\right) \Phi_{S}\left(\mathbf{R}^{-}\right)+\Phi_{S}\left(\mathbf{R}^{-}\right) \Phi_{G}^{-}\left(\mathbf{R}^{+}\right) .
\end{aligned}
$$

The parameters $g_{k}$ are adjusted to achieve stability and minimize the variance of the fermion energy.

Note that $\Phi^{ \pm}{ }_{\mathrm{G}}$ and $\Psi$ have cusps at the nodes of $\varphi_{\mathrm{A}}(\mathbf{R})$. An alternative to $\Phi^{ \pm}{ }_{\mathrm{G}}$ with no cusps is

$$
\begin{aligned}
\Phi_{G}^{ \pm}(\mathbf{R})= & \sqrt{\varphi_{S}^{2}(\mathbf{R})+g_{1}^{2} \varphi_{A}^{2}(\mathbf{R})} \pm g_{0} \varphi_{A}(\mathbf{R}) \\
& +g_{0} \sqrt{\varphi_{A}^{2}(\mathbf{R})+g_{2}^{2}\left(\nabla \varphi_{A}(\mathbf{R})\right)^{2}},
\end{aligned}
$$

where $\nabla \varphi_{A}(\mathbf{R})$ measures the change of $\varphi_{A}(\mathbf{R})$ needed to smooth the cusp.

The following properties hold for any an odd permutation $P$.

$$
\begin{gathered}
\Phi_{G}^{ \pm}(P \mathbf{R})=\Phi_{G}^{ \pm}(\mathbf{R}) \\
\Psi\left(P \mathbf{R}^{+}, P \mathbf{R}^{-}\right)=\Psi\left(\mathbf{R}^{-}, \mathbf{R}^{+}\right) \\
\Psi\left(P \mathbf{R}^{+}, P \mathbf{R}^{-}\right) \neq \Psi\left(\mathbf{R}^{+}, \mathbf{R}^{-}\right) .
\end{gathered}
$$

If $\mathbf{R}_{\mathbf{0}}^{+}, \mathbf{R}_{\mathbf{0}}^{-}$are such that

$$
\begin{aligned}
& \varphi_{A}\left(\mathbf{R}_{0}^{+}\right)>0 \\
& \varphi_{A}\left(\mathbf{R}_{0}^{-}\right)<0
\end{aligned}
$$


then

$$
\begin{aligned}
& \Psi\left(\mathbf{R}_{0}^{+}, \mathbf{R}_{0}^{-}\right)>\Psi\left(P \mathbf{R}_{0}^{+}, \mathbf{R}_{0}^{-}\right)>\Psi\left(P \mathbf{R}_{0}^{+}, P \mathbf{R}_{0}^{-}\right), \\
& \Psi\left(\mathbf{R}_{0}^{+}, \mathbf{R}_{0}^{-}\right)>\Psi\left(\mathbf{R}_{0}^{+}, P \mathbf{R}_{0}^{-}\right)>\Psi\left(P \mathbf{R}_{0}^{+}, P \mathbf{R}_{0}^{-}\right) .
\end{aligned}
$$

These last inequalities state that if both the positive and negative walkers are in their favorable "pockets," then the importance functions $\Psi$ are decreased when odd permutations are applied to either or both sets of coordinates in either order.

In practice, the function $\Psi$ was modified further to reflect the fact that cancellation of close walkers means that the importance must vanish as they approach each other. Consideration of the diffusion process with cancellation suggests the following form:

$$
\Psi_{C}\left(\mathbf{R}^{+}, \mathbf{R}^{-}\right)=\Psi\left(\mathbf{R}^{+}, \mathbf{R}^{-}\right) \operatorname{erf}\left(g_{3} k_{F} /(2 \pi)\left|\mathbf{R}^{+}-\mathbf{R}^{-}\right|\right) .
$$

As indicated above, in the computations reported here, the drift and branching for the basic diffusion Monte Carlo was carried out using the symmetric trial function $\varphi_{\mathrm{S}}(\mathbf{R})$. This takes care of cusps associated with the physical potentials - here Coulomb potentials. Then after a pair of walkers is advanced by means of drift, correlated diffusion, symmetric branching, cancellation, and " repairing," the new pairs are branched again on the basis of the ratio of $\Psi_{\mathrm{C}}$ before and after.

It is useful to review here the technique involved in correlating the two walkers. The diffusion step for any walker requires generating a $3 \mathrm{~N}$ dimensional vector $\mathbf{U}^{+}$whose components are drawn independently from a Gaussian distribution of mean zero and variance $\delta \tau$, the increment in imaginary time. That is, a positive walker is moved from $\mathbf{S}^{+}$to $\mathbf{R}^{+}$by

$$
\mathbf{R}^{+}=\mathbf{S}^{+}+\mathbf{U}^{+}
$$

and a negative walker by

where

$$
\mathbf{R}^{-}=\mathbf{S}^{-}+\mathbf{U}^{-},
$$




$$
\mathbf{U}^{-}=\mathbf{U}^{+}-2\left[\frac{\mathbf{S}^{+}-\mathbf{S}^{-}}{\left|\mathbf{S}^{+}-\mathbf{S}^{-}\right|} \cdot \mathbf{U}^{+}\right] \frac{\mathbf{S}^{+}-\mathbf{S}^{-}}{\left|\mathbf{S}^{+}-\mathbf{S}^{-}\right|}
$$

\section{Acceptance/Rejection Dynamics}

We review here a few basics of DMC dynamics and show how it can be generalized to take into account acceptance/rejection, correlated diffusion, and cancellation. We use the simple unsymmetric short-time propagator

$$
G_{0}(\mathbf{R}, \mathbf{S} ; \ddot{a} \tau)=\frac{\left.\exp \left\lfloor-\left(\mathbf{R}-\mathbf{S}-\ddot{a} o \nabla \varphi_{S}(\mathbf{S})\right) / \varphi_{S}(\mathbf{S})\right)^{2} / 2 \ddot{a} \hat{o}\right\rfloor}{(2 \partial \ddot{a} \hat{o})^{3 N / 2}}
$$

and define

$$
\begin{aligned}
& E_{L}(\mathbf{R})=\frac{H \varphi_{S}(\mathbf{S})}{\varphi_{S}(\mathbf{S})} \\
& B(\mathbf{R})=e^{\left[E_{T}-E_{L}(\mathbf{R})\right] \delta \tau} \\
& G(\mathbf{R}, \mathbf{S} ; \delta \tau)=B(\mathbf{R}) G_{0}(\mathbf{R}, \mathbf{S} ; \delta \tau) .
\end{aligned}
$$

Cancellation of walkers means that the algebraic density of walkers of opposite signs that is generated by means of $\mathrm{G}_{0}$ can be canceled if they are close enough. When importance sampling is used, what is cancelled is the density unweighted by the importance function. So a pair of walkers $\left(\mathbf{R}_{\mathbf{k}}^{+}, \mathbf{R}_{\mathbf{k}}{ }^{-}\right)$ generates an unweighted net density at $\mathbf{R}$ given by

$$
\begin{aligned}
& \psi_{F}(\mathbf{R}, \tau+\delta \tau)= \\
& \frac{\left.B(\mathbf{R}) G_{0}\left(\mathbf{R}, \mathbf{R}_{k}^{+} ; \delta \tau\right)\right)}{\varphi_{S}(\mathbf{R})}-\frac{\left.B(\mathbf{R}) G_{0}\left(\mathbf{R}, \mathbf{R}_{k}^{-} ; \delta \tau\right)\right)}{\varphi_{S}(\mathbf{R})} .
\end{aligned}
$$

Using "acceptance/rejection" means replacing the propagator G by 


$$
\begin{aligned}
G_{A R}(\mathbf{R}, \mathbf{S} ; \delta \tau) & =A(\mathbf{R}, \mathbf{S}) B(\mathbf{R}) G_{0}(\mathbf{R}, \mathbf{S} ; \delta \tau) \\
& +p_{r}(\mathbf{S}) B(\mathbf{S}) \delta(\mathbf{R}-\mathbf{S})
\end{aligned}
$$

where $\mathrm{A}(\mathbf{R}, \mathbf{S})$ is the probability that a move proposed from $\mathbf{S}$ to $\mathbf{R}$ is accepted, and $p_{r}$ is the total probability that moves from $\mathbf{S}$ are rejected. We separate positive and negative parts of the density by applying the identity

$$
x=\max (x, 0)-\max (-x, 0) .
$$

We find the following propagators for the positive and negative parts of the future density:

$$
\begin{aligned}
G_{C A R}^{+}\left(\mathbf{R}, \mathbf{R}_{k}^{+} ; \delta \tau\right) & =A\left(\mathbf{R}, \mathbf{R}_{k}^{+}\right) B(\mathbf{R}) G_{0}\left(\mathbf{R}, \mathbf{R}_{k}^{+} ; \delta \tau\right) \\
& \times \max \left(1-\frac{A\left(\mathbf{R}, \mathbf{R}_{k}^{-}\right) G_{0}\left(\mathbf{R}, \mathbf{R}_{k}^{-} ; \delta \tau\right)}{A\left(\mathbf{R}, \mathbf{R}_{k}^{+}\right) G_{0}\left(\mathbf{R}, \mathbf{R}_{k}^{+} ; \delta \tau\right)}, 0\right) \\
& +p_{r}\left(\mathbf{R}_{k}^{+}\right) B\left(\mathbf{R}_{k}^{+}\right) \delta\left(\mathbf{R}-\mathbf{R}_{k}^{+}\right) ; \\
G_{C A R}^{-}\left(\mathbf{R}, \mathbf{R}_{k}^{-} ; \delta \tau\right) & =A\left(\mathbf{R}, \mathbf{R}_{k}^{-}\right) B(\mathbf{R}) G_{0}\left(\mathbf{R}, \mathbf{R}_{k}^{-} ; \delta \tau\right) \\
& \times \max \left(1-\frac{A\left(\mathbf{R}, \mathbf{R}_{k}^{+}\right) G_{0}\left(\mathbf{R}, \mathbf{R}_{k}^{+} ; \delta \tau\right)}{A\left(\mathbf{R}, \mathbf{R}_{k}^{-}\right) G_{0}\left(\mathbf{R}, \mathbf{R}_{k}^{-} ; \delta \tau\right)}, 0\right) \\
& +p_{r}\left(\mathbf{R}_{k}^{-}\right) B\left(\mathbf{R}{ }_{k}^{-}\right) \delta\left(\mathbf{R}-\mathbf{R} \mathbf{R}_{k}^{-}\right) .
\end{aligned}
$$




\section{Results for the two-dimensional electron gas}

We have carried out a set of computations for the two-dimensional electron gas at $r_{\mathrm{S}}=1$. The symmetric trial function, $\varphi_{\mathrm{S}}(\mathbf{R})$, was simply an electronelectron Jastrow product, and the antisymmetric function, $\varphi_{A}(\mathbf{R})$, was $\varphi_{S}(\mathbf{R})$ times a Slater determinant of plane waves. Recall that in the present form of our methods, there can be linear dependence in time step and noticeable dependence on population size, so that a substantial computing effort is required. Results are summarized in Table 1 and compared with a calculation by Kwon, Ceperley, and Martin $^{4}$ (which included backflow in the determinant basis functions).

Table 1. Energy per particle for the two-dimensional electron gas at $\mathbf{r}_{\mathbf{s}}=1$

\begin{tabular}{cccc}
\hline $\mathrm{N}_{\mathrm{e}}$ & FMC(1) & FMC(2) & DMC \\
\hline 10 & $-0.4582(10)$ & & \\
26 & $-0.3910(17)$ & $-0.3908(17)$ & $-0.3910(12)^{*}$ \\
\hline & & $*$ Released node $^{4}$
\end{tabular}

FMC(1) refers to a set of calculations with importance functions with cusps; FMC(2) to a set without cusps. The fact that these agree with each other and with the reliable released node computation ${ }^{4}$ is a strong verification of the correctness of our method.

Table 2. Efficiency vs. electron number

\begin{tabular}{ccc}
\hline $\mathrm{N}_{\mathrm{e}}$ & Computing time per step* & Figure of merit* \\
\hline 10 & 0.018 & 0.0077 \\
26 & 0.101 & 0.016 \\
\hline
\end{tabular}

* Arbitrary relative units

In Table 2, we show the decline in the computational efficiency as electron number, increases from 10 to 26 . The "figure of merit" is the product of the variance of a typical run by the aggregate computing time for that run. It is notable that this measure of efficiency decreases only by a factor of two as the electron number doubles. Preliminary results show a modest increase for 58 electrons; we are in the process of revising our programs to treat larger electron systems even more efficiently. 


\section{Acknowledgements}

This work was performed under the auspices of the U.S. Department of Energy by the University of California, Lawrence Livermore National Laboratory under contract No. W-7405-Eng.-48.

\section{References}

1. P.J. Reynolds, D.M. Ceperley, B.J. Alder, and W.A. Lester, Jr., J. Chem. Phys. 77, 5593 (1982)

2. J.B. Anderson, J. Chem. Phys. 63, 1499 (1975)

3. M. H. Kalos and Francesco Pederiva, Phys. Rev. Lett. 85, 3457 (2000)

4. Y. Kwon, D..M. Ceperley, and R.M. Martin, Phys. Rev. B. 53, 7376 (1996) 